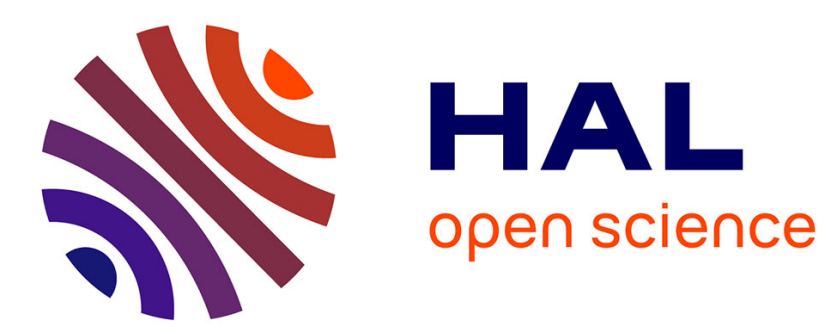

\title{
Optical performance and laser induced damage threshold improvement of diffraction gratings used as compressors in ultra high intensity lasers
}

Jerome Neauport, Bonod Nicolas

\section{- To cite this version:}

Jerome Neauport, Bonod Nicolas. Optical performance and laser induced damage threshold improvement of diffraction gratings used as compressors in ultra high intensity lasers. Optics Communications, 2005, pp.649-655. 10.1016/j.optcom.2005.10.069 . cea-01053381

HAL Id: cea-01053381

https://hal-cea.archives-ouvertes.fr/cea-01053381

Submitted on 30 Jul 2014

HAL is a multi-disciplinary open access archive for the deposit and dissemination of scientific research documents, whether they are published or not. The documents may come from teaching and research institutions in France or abroad, or from public or private research centers.
L'archive ouverte pluridisciplinaire HAL, est destinée au dépôt et à la diffusion de documents scientifiques de niveau recherche, publiés ou non, émanant des établissements d'enseignement et de recherche français ou étrangers, des laboratoires publics ou privés. 


\title{
Optical performance and laser induced damage threshold improvement of diffraction gratings used as compressors in ultra high intensity lasers
}

\author{
Nicolas Bonod *, Jérôme Néauport \\ Commissariat à l'Energie Atomique, Centre d'Etudes Scientifiques et Techniques d'Aquitaine, BP2, F-33114 Le Barp, France
}

Received 22 July 2005; received in revised form 26 October 2005; accepted 27 October 2005

\begin{abstract}
This paper studies gratings engraved in a multilayer dielectric stack for ultra high intensity laser compressors. A metal layer is inserted between the substrate and the dielectric stack to reduce the number of dielectric bilayers and thus the mechanical stress within the stack. A code taking account the fluctuation range of the geometrical parameters during the fabrication process is used to numerically optimize the mirror stack and study different groove profiles to increase the reflected efficiency and the laser induced damage threshold. It is evidenced that of all the profiles leading to good diffraction performances, those with the greatest groove depth and width values result in the smallest enhancement of the electric field square inside the grating with a decrease by a factor close to 2.5 .
\end{abstract}

(C) 2005 Elsevier B.V. All rights reserved.

\section{Introduction}

A Petawatt laser is currently being developed in the laser integration line (LIL), the prototype of the future Megajoule Laser, in France [1]. This Petawatt laser line is designed to deliver impulses of 7.2 PW in $500 \mathrm{fs}$ at the wavelength of $1053 \mathrm{~nm}$. The chirped pulse amplification (CPA) method will be used [2]. We are working on the modeling and the fabrication of the gratings used in the CPA scheme. The compression stage uses several gratings [1]. In their design, emphasis is given, respectively, to the diffraction efficiency performance levels for the first gratings and to the laser damage threshold for the last one. The objective for this last grating is to build a component operating at $77.2^{\circ}$ with 1780 $\mathrm{g} / \mathrm{mm}$ and reflecting more than $95 \%$ of the incident light into the -1 order inside a spectral band of $6 \mathrm{~nm}$ centered at the wavelength of $1053 \mathrm{~nm}$. This grating must present a damage

\footnotetext{
* Corresponding author. Address: Institut Fresnel, CLARTE, UMR 6133 Faculte des Sciences et Techniques, 13397 Marseille, France. Tel.: +33 491288824; fax: +33 491674428 .

E-mail addresses: nicolas.bonod@fresnel.fr, nicolas.bonod@cea.fr (N. Bonod).
}

threshold higher than $3 \mathrm{~J} / \mathrm{cm}^{2}$ in the right section in a vacuum environment. For that purpose, it is now well known that metal gratings, usually made of gold, must be replaced by multilayer dielectric ones (MLD) [3-7]. These dielectric gratings appeared in the middle of the 1990s and considerably increased the laser induced damage threshold. This study is dedicated to the design of the grating structure with the best performance giving a high reflectivity in the -1 order and the lowest field enhancement in the material and hence the highest damage threshold [7-9]. MLD gratings, composed of a multi-dielectric mirror with its top layer engraved, can exhibit crazing when operating in a vacuum environment [10]. The probability of crazing increases with the number of layers. An original solution using a metal insert between the substrate and the multi-dielectric mirror stack is therefore studied to reduce the stack thickness while preserving its optical properties and damage threshold.

Section 2 provides a description of pulse compression gratings with an overview of the different devices able to reflect practically all the incident light in the -1 order. Section 3 explains the numerical method developed to select the best profiles. Based on the rigorous differential method, it is capable of accurately resolving Maxwell equations in 
periodic devices with a trapezoidal modulation. The main difficulty of a numerical study is to provide profiles that can be reproduced by the constructor and for which the performance levels of the manufactured grating are equal to those of the model. Increment steps and parameter domains were therefore adjusted to correspond to manufacturing capabilities. Section 4 is dedicated to the design of the dielectric stack, showing the value of a metal insertion for reducing the number of dielectric bilayers. Finally, Section 5 is dedicated to the design of the grating profile. The evolution of the maximum of the electric field norm inside the solid material of the grating is studied as a function of the profile geometry. This study is important for the laser damage threshold of the grating. It will be shown that among the profiles selected with respect to the diffraction criteria, the maximum of the square of the electric field value can be reduced by a factor of about 2.5 .

\section{Overview of pulse compression gratings}

It is well known that a metal grating can reflect almost all the incident light in the $(-1)$ th order [11]. The metal substrate reflects the incident light and a suitable groove profile diffracts the reflected light into the $(-1)$ th order. To increase the laser induced damage threshold, however, a grating with dielectric materials must be built [3-6] Hence, a dielectric device which reflects all the incident light must first be manufactured. To that end, the total internal reflection conditions must be fulfilled [12]. An initial solution consists of using an antireflection dielectric coating deposited on a dielectric material (fused silica, for example) while the substrate is air. If the total internal reflection condition is satisfied, all the incident light is reflected. The interface where the reflection occurs is then engraved in such a way that only the (0)th and the $(-1)$ th orders can propagate $[13,14]$. Unfortunately, nonlinear effects in the dielectric material make this solution unsuitable for the compression of petawatt pulses. To reduce the nonlinear effects, the superstrate must be made of air. The only way to produce a total internal reflection is to find a substrate with an optical index of less than 1 . Such a material cannot be found in nature but it is possible to build a dielectric device such as a photonic crystal for which the homogenization limit, under optogeometrical conditions, provides an optical index of less than 1. For that purpose, identical bilayers made of two dielectric materials with a high optical index $n_{\mathrm{H}}$ and a low optical index $n_{\mathrm{L}}$ are deposited onto a substrate. A high number of pairs of layers improves the reflection coefficient of the stack and thus the reflected efficiency in the -1 order of the final MLD grating. Unfortunately, it also gives rise to induced stress within the stack which can result in crazing after exposure to a vacuum environment. Then, the grating may be distorted making it difficult to obtain a good wavefront quality. This paper therefore studies the possibility of partially replacing the dielectric substrate by a metal one to reduce the number of pairs. The metal must present a high conductivity, like gold or silver, to reflect the light with low losses. Silver is not suitable since it exhibits too high a reflectivity at the wavelength used during the holographic recording of the photoresist material. Thanks to its low reflectivity, gold can be used at this wavelength. There must, however, be a sufficient number of pairs for an adequate reduction of the electric field at the dielectric-metal interface to maintain a high damage threshold. In the manufacturing process, a thick metal layer can be deposited on a dielectric substrate, and the bilayers then deposited on the metal layer. Numerically, the dielectric substrate is replaced by the metal one.

In order to reflect more than $95 \%$ of the incident light in the $(-1)$ th order, the top layer is then engraved in such a way that the $(0)$ th and the $(-1)$ th orders can propagate. It has been evidenced that the etching must be performed in the material with the lowest optical index to increase the laser damage threshold [15]. The etching process makes the geometry of the grooves trapezoidal with a slope angle $\alpha$ of nearly $87.5^{\circ}$.

\section{Optimization of the grating performance levels}

The grating performance levels are numerically optimized firstly as a function of the 4 parameters $e, h, c$ and $\alpha$ (see Fig. 1). A numerical code has been developed to calculate the reflected efficiency in the $(-1)$ th order for each combination $\{e, h, c, \alpha\}$ and for 3 different couples $(\lambda, \theta)$, around $1053 \mathrm{~nm}$ and $77.2^{\circ}$. The differential method is used. It is able to resolve Maxwell equations in gratings presenting a trapezoidal modulation [16]. With this method, each spatial electromagnetic field component is developed onto $2 N+1$ Fourier components. The convergence of the method with respect to $N$ depends on the optical index contrast of materials inside the modulated area. In our case, dielectric materials are involved with the result that $N$ is taken equal to 4 to calculate the efficiencies and equal to 10 to plot the electric field map.

The manufacturer defines the range of each variable $\{e$, $h, c, \alpha\}$. For example, the range of $c$ decreases when $h$ increases. More precisely, the minimum value of $c$ increases when $h$ increases. Moreover, the constructor cannot engrave and measure the grating profile with high accuracy. The increment steps are taken equal to half of the fluctuation ranges $\Delta e, \Delta h, \Delta c, \Delta \alpha$ of each variable estimated by the constructor. The code provides the average of the set of efficiencies inside the fluctuation range. This value will be taken in what follows as being the only physical value representative of the real performance levels of the grating after fabrication.

Optimization is performed as follows:

Step 1: If the reflected efficiency in the $(-1)$ th order is higher than $90 \%$ for $\lambda$ equal to 1050 and $1056 \mathrm{~nm}$, and higher than $95 \%$ for $\lambda$ equal to $1053 \mathrm{~nm}$, the combination $\{e, h, c, \alpha\}$ is stored. At this step, it would be easy to select the combination providing the highest efficiency. Unfortunately, the constructor is unable to build a grating with the 


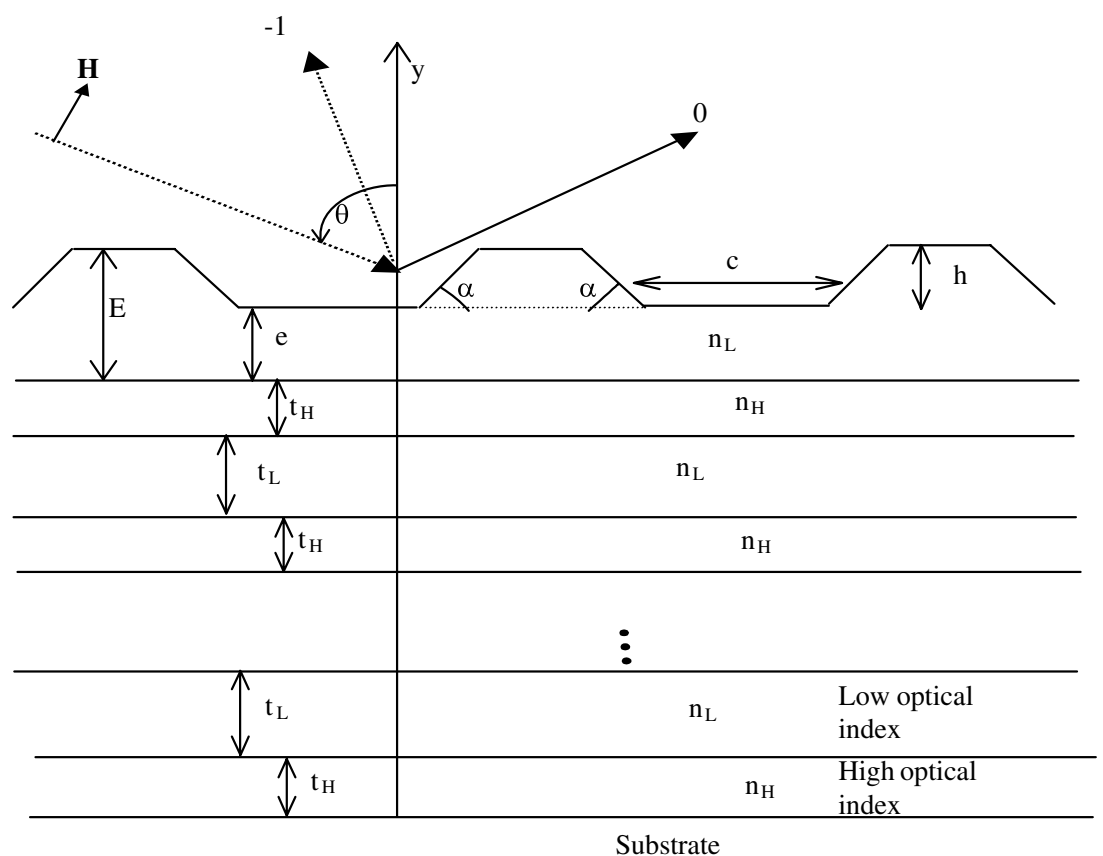

Fig. 1. Substrate of glass $(n=1.46) . t_{\mathrm{H}}$ is the layer of the high optical index $\left(n_{\mathrm{H}}=1.9\right)$ medium; $t_{\mathrm{L}}$ the layer of the low optical index $\left(n_{\mathrm{L}}=1.46\right)$ medium; $\theta$ the angle of incidence of the transverse electric (TE) polarized incident plane wave. Gratings present a trapezoidal geometry with a slope angle $\alpha$ and a period $d$. The thickness of the grooves is $h$ and the groove width defined at the half depth is $c$.

exact values of $e, h, c$, and $\alpha$. Consequently, the performance levels of the manufactured grating may differ considerably from those of the modeled one. To overcome this difficulty, in step 2 the average of the efficiencies with different values of $e, h, c$, and $\alpha$ inside their fluctuation range will be calculated.

Step 2: As the increment steps are taken equal to half of the fluctuation ranges, the ranges of the geometrical variables are:

$$
\begin{aligned}
& {[\alpha-\Delta \alpha, \alpha+\Delta \alpha],[h-\Delta h, h+\Delta h],} \\
& \quad[c-\Delta c, c+\Delta c],[e-\Delta e, e+\Delta e] .
\end{aligned}
$$

For each combination $\{e, h, c, \alpha\}$, a sample mean of the efficiency is provided with the calculation of the 81 efficiencies of the following combinations:

$$
\begin{aligned}
& \{\alpha+w \Delta \alpha, h+x \Delta h, c+y \Delta c, e+z \Delta e\}, \\
& \quad w, x, y, z=[-1,0,1] .
\end{aligned}
$$

As the thicknesses of the plane layers are assumed to be correctly reproduced, the relations:

$h+e=\mathbf{E}$,

$x+z=0$

are obtained so that the number of combinations of the set can be reduced to 27 . The combinations $\{e, h, c, \alpha\}$ giving averaged efficiencies higher than $95 \%$ are selected. In summary, for the total number of combinations $\{e, h, c, \alpha\}$, two selections are operated. The first concerns the performance of the grating in the spectral band whilst the second concerns the stability of the performance levels in the fluctua- tion ranges of the geometrical parameters determined by the fabrication process. The value of the slope angle $\alpha$ is very difficult to measure when it is higher than $85^{\circ}$ and depends on the ion etching technique but inverse methods and atomic force microscopy observations tend towards the value $\alpha=87.5^{\circ}$ with $\Delta \alpha=1^{\circ}$. The precision ranges of the thicknesses $h$ and $e$ depend on the values of $h$ and $e$ and have been chosen equal to $\Delta h=0.1 h$ and $\Delta e=0.1 e$. As far as $\Delta c$ is concerned, this depends on the period $d$, and is taken equal to $\Delta c=0.1 d$.

It has been shown that the value of the electric field norm in the material determines the laser damage threshold [7-9]. Consequently, after the two successive selections, for each selected combination $\{e, h, c, \alpha\}$, the maximum of the electric field norm is calculated inside the solid material, and not inside the grooves, made of air. The geometry of modulation producing the smallest enhancement of the electric field inside the grating is then selected.

\section{Design of the dielectric mirror}

\subsection{Determination of the layer thicknesses}

Thicknesses $t_{\mathrm{H}}$ and $t_{\mathrm{L}}$ of high and low optical index materials $n_{\mathrm{H}}$ and $n_{\mathrm{L}}$ of a $(\lambda / 4 ; \lambda / 4)$ coating are equal to

$t_{\mathrm{H}}=\frac{\lambda}{4 n_{\mathrm{H}} \cos \theta_{\mathrm{H}}}, \quad t_{\mathrm{L}}=\frac{\lambda}{4 n_{\mathrm{L}} \cos \theta_{\mathrm{L}}}$,

where $\theta_{\mathrm{H}}$ and $\theta_{\mathrm{L}}$ are the angles of the refracted plane wave in high and low optical index materials, respectively, calculated with the formula 


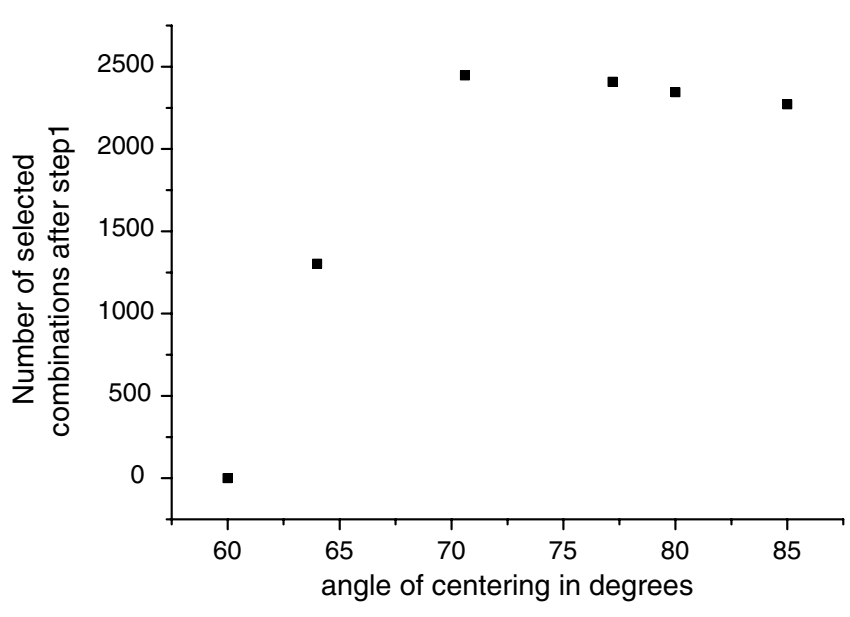

Fig. 2. Number of combinations $\{e, h, c, \alpha\}$ selected after step 1 as a function of the angle of centering $\theta_{\mathrm{i}}$. Calculations are made with 9 bilayers.

$\theta_{\mathrm{H}}=\sin ^{-1}\left(\frac{\sin \theta_{\mathrm{i}}}{n_{\mathrm{H}}}\right), \quad \theta_{\mathrm{L}}=\sin ^{-1}\left(\frac{\sin \theta_{\mathrm{i}}}{n_{\mathrm{L}}}\right)$,

where $\theta_{\mathrm{i}}$ is the angle at which the stack is centered. It must be recalled that $\theta_{\mathrm{i}}$ is not known because the mirror is placed below the grating, and must reflect the 0 and the -1 transmitted orders by the grating. Consequently, $\theta_{\mathrm{i}}$ is not equal to the angle of incidence of light on the grating $\theta$. In Fig. 2, the number of combinations selected after step 1 is presented as a function of the angle of centering (see Fig. 1). In other words, to determine the angle $\theta_{\mathrm{i}}$ to implement in Eq. (1), the number of combinations at the end of step 1 is calculated for different mirrors designed at different angles of centering $\theta_{\mathrm{i}}$ (Fig. 2) among which the following values:

$\theta_{\mathrm{i}}=\theta=77.2^{\circ}$

$\theta_{\mathrm{i}}=\sin ^{-1}(\sin \theta-\lambda / d)=64.0^{\circ}$,

$\theta_{\mathrm{i}}=\theta_{\mathrm{m}}=\frac{\theta+\sin ^{-1}(\sin \theta-\lambda / d)}{2}=70.6^{\circ}$

correspond, respectively, to the 0 order, to the median angle between the -1 and 0 orders, and to the -1 order. It can be seen that the stack denoted 2, optimized to reflect the light at an incidence corresponding to the median angle between the $(0)$ th and the $(-1)$ th order, gives the highest number of solutions. This result was expected because the stack must reflect the (0)th and the (-1)th transmitted orders. In what follows, the numerical studies will be made with this last configuration $\left(70.6^{\circ}\right)$.

\subsection{Value of a metal substrate}

To evidence the value of the gold insertion, the curves in Fig. 3 compare the number of solutions after the 2 successive selections for dielectric and metal substrates as a function of the number of bilayers. The convergence of the curves is better after step 1, compared to step 2, especially with the use of a metal substrate. It shows that the number of dielectric bilayers is crucial for the stability of the grating performance levels inside the fluctuation range. In Fig. 3(a),
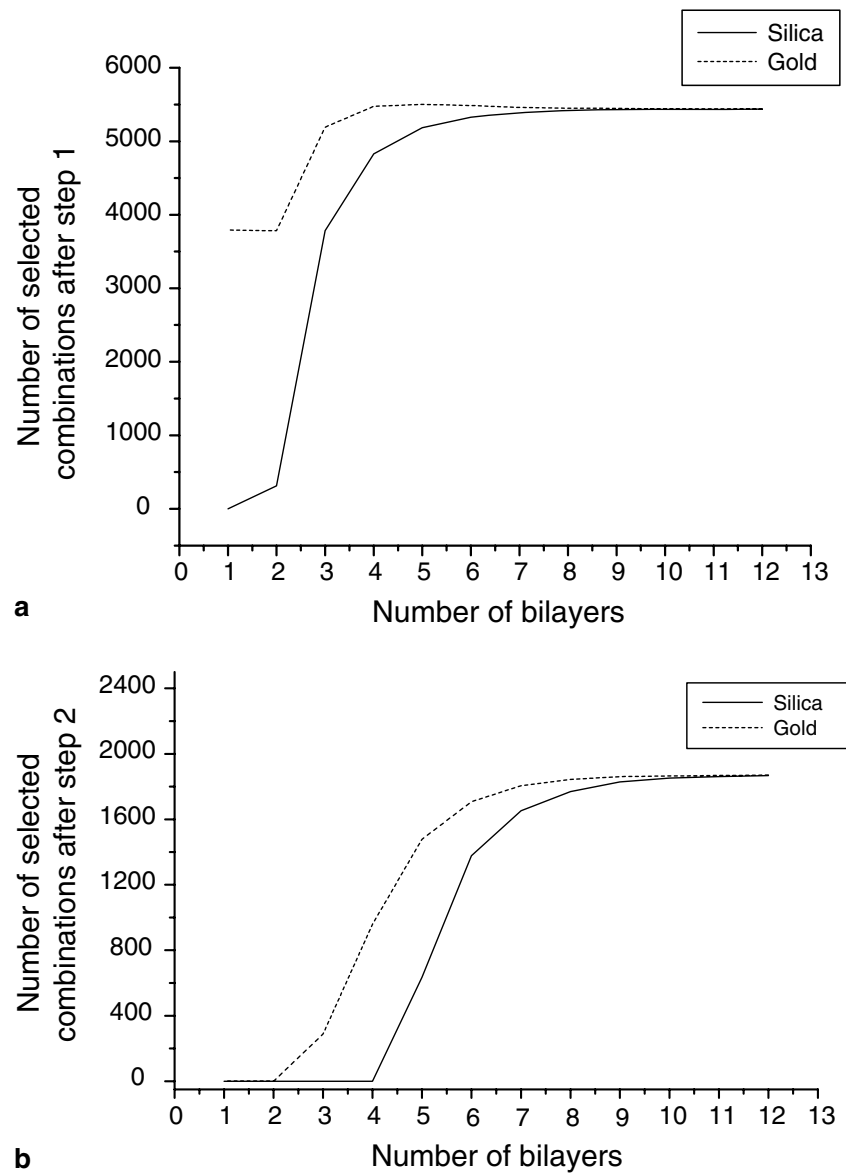

Fig. 3. Number of combinations $\{e, h, c, \alpha\}$ selected after steps 1 (a) and 2 (b) as a function of the number of dielectric bilayers. Solid line: substrate in silica, dashed line: substrate in gold.

it can be seen that 4 bilayers are sufficient with the use of a metal substrate whilst 8 or 9 bilayers are needed to obtain the same performance levels with a dielectric substrate. The same phenomenon is observed after step 2, where 7 bilayers with a metal substrate produce the same performance levels as 9 bilayers deposited on a dielectric substrate. This study shows the high value of a metal substrate for the decrease of the number of dielectric bilayers needed in a multilayer dielectric grating and thus for the decrease of the mechanical stress inside the stack. The entire study will now therefore be made with 7 bilayers deposited on a metal substrate.

\section{Design of the grating profile}

\subsection{Evolution of the number of combinations as a function of $\alpha$}

In this section, the performance levels of the grating are studied as a function of the slope angle $\alpha$. Results in Fig. 4 show that the number of combinations selected after steps 1 and 2 is very sensitive to $\alpha$ and regularly increases with it to reach its maximum at $\alpha=90^{\circ}$. This is an important result, since even if manufacturers have difficulty in 


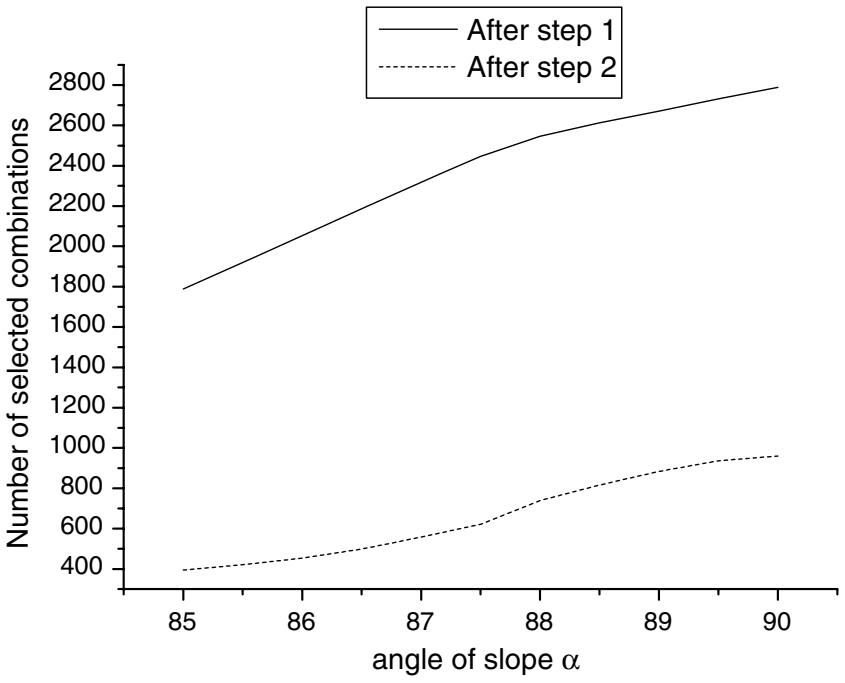

Fig. 4. Number of selected combinations as a function of the slope angle $\alpha$ of the pillars.

measuring the slope angle when it exceeds $85^{\circ}$, it is clearly shown that lamellar profiles with slope angles equal to $90^{\circ}$ give gratings with the best performance.

As explained in Section 2, from observations made on manufactured gratings a slope angle $\alpha$ equal to $87.5 \pm$ $0.5^{\circ}$ is retained for which 621 combinations are selected after step 2 (see Fig. 4). The aim of the following study is to select from those 621 combinations the best profile with respect, first, to the reflected efficiency (Section 5.2) and, second, to the laser damage threshold (Section 5.3).

\subsection{Evolution of the reflected efficiency as a function of the grating profile}

The gratings studied present a very large surface area $(495 \mathrm{~mm} \times 900 \mathrm{~mm})$ with the result that their profiles and therefore their optical properties may fluctuate along the surface. Gratings must present similar properties along the surface to preserve the homogeneity of the laser beam. To model this property, it is assumed that each grating profile $\{e, h, c, \alpha\}$ can be engraved uniformly following 27 geometrical parameters (as explained in Section 3) over the whole surface of the grating. With this set of profiles, it is possible to calculate the average $\bar{x}$ and the variance of efficiency calculated with the well known formula $\sum_{i=1}^{27}\left(x_{i}-\bar{x}\right)^{2} / 27$, where $x_{i}$ is the efficiency of the $(i)$ th profile. As the grating is engraved uniformly following 27 geometrical parameters, $\bar{x}$ can be related to the averaged efficiency over the whole surface of the grating and must be increased to its maximum whilst the variance must be decreased to preserve the homogeneity of the laser beam. Fig. 5 are plots of the efficiency means calculated for each profile, as a function of the geometrical parameters $h$ (Fig. 5(a)) and $c$ (Fig. 5(b)). The values of the groove thickness $h$ do not exceed $700 \mathrm{~nm}$ (Fig. 5(a)). Moreover, Fig. 4(b) shows a high sensitivity of the mean efficiency with respect to $c$. Indeed, from its maximum at $c=394 \mathrm{~nm}$, the mean
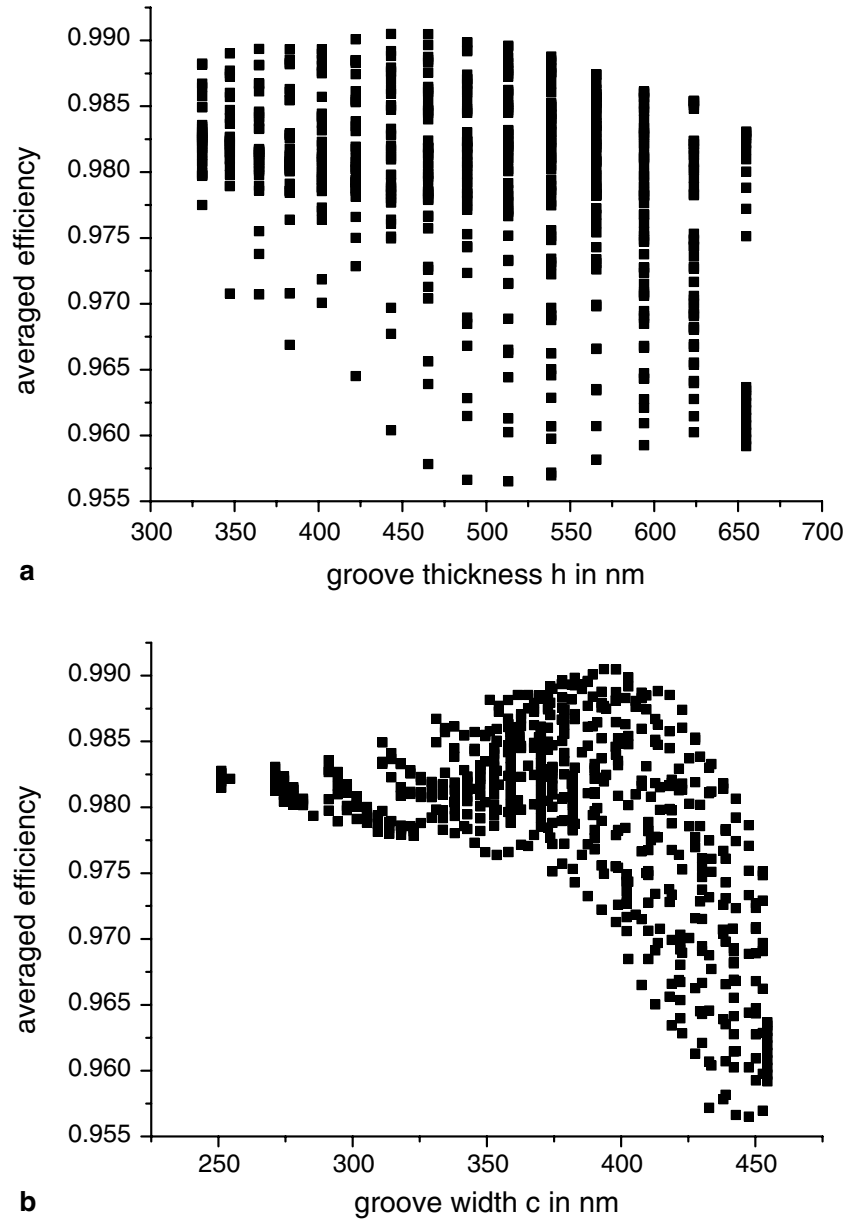

Fig. 5. Plot of the average of the 27 reflected efficiencies, calculated in the uncertainty range of the 4 geometrical parameters, as a function of $h$ and c. Averages higher than $99 \%$ can be obtained.

efficiency decreases considerably for higher values of $c$ to reach its minimum at $c=450 \mathrm{~nm}$. The most important conclusion, however, is that with a metal insertion and only 7 bilayers, it is possible to build gratings capable of presenting a sample mean of efficiency over the whole surface of the grating higher than $99 \%$. The maximum is obtained with $h=443 \mathrm{~nm}$ and $c=394 \mathrm{~nm}$.

\subsection{Evolution of the maximum of the electric field norm inside the material as a function of the grating profile}

To increase the laser damage threshold of the grating, a maximum reduction of the enhancement of the electric field inside the material must be obtained. In Fig. 6, the maximum of the electric field norm square $|E|^{2}$ in the material is plotted as a function of the parameters $h$ and $c$ (the amplitude of the incident electric field is taken to be equal to unity). It can be observed in both figures that the highest values of $h$ and $c$ produce the smallest enhancement of the electric field inside the grating. The high dependence of the groove width value on the electric field enhancement can be very well observed in Fig. 6(b). Moreover, Section 4.2 evidenced that the slope angle must be increased up to $90^{\circ}$ to give a high number of 

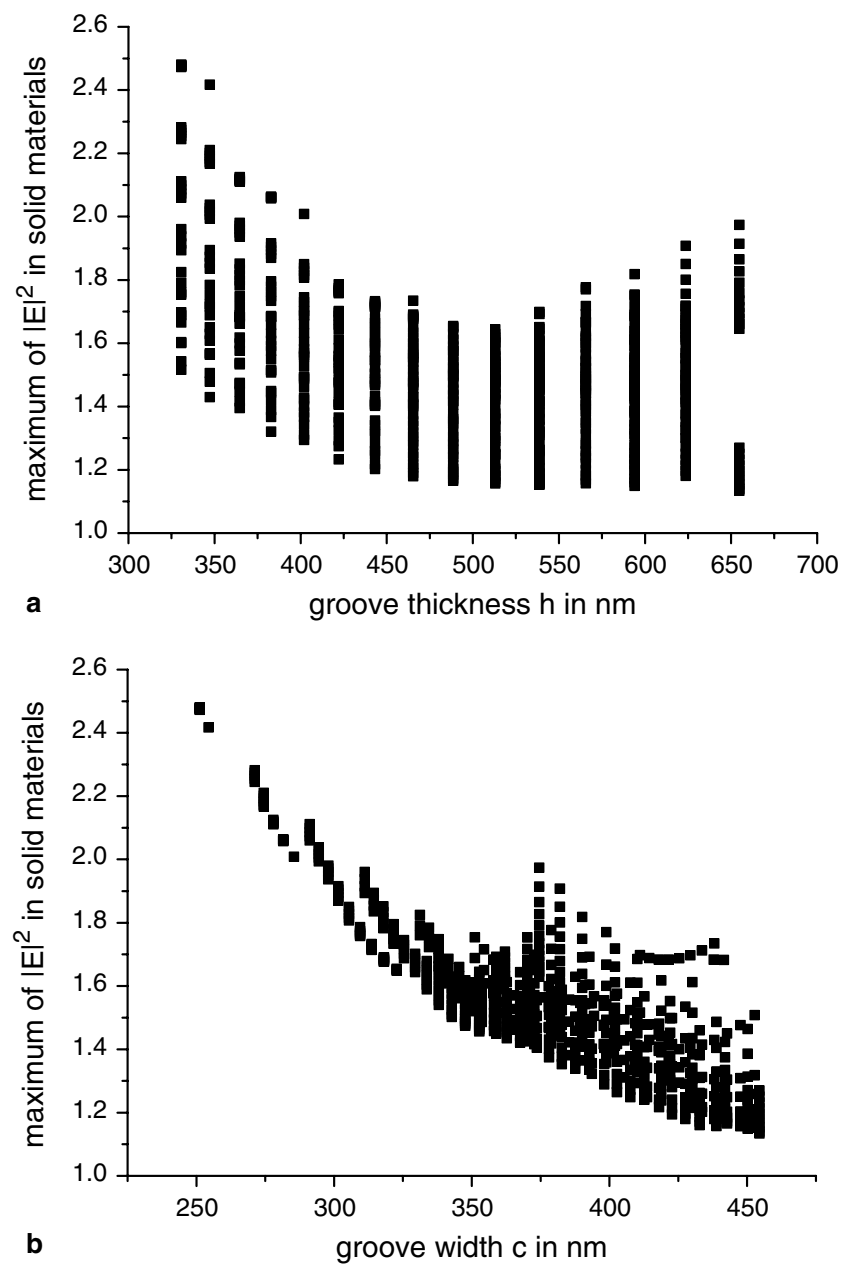

Fig. 6. Maximum of the enhancement of $|E|^{2}$ in the solid materials as a function of the geometrical parameters $h$ (a) and $c(\mathrm{~b})$.

selected profiles after step 2 . The necessary increase of the groove thickness is also a positive factor because during the manufacturing process the thicker the groove the higher the slope angle. Nevertheless, it is more difficult to build deep gratings with a high quality of etching. Consequently, compared to a grating with a groove thickness of $300 \mathrm{~nm}$, a grating with a groove thickness twice that size may present more defects resulting in local field enhancements and reducing the laser damage threshold. However, a reduction of $|E|^{2}$ by a factor close to 2.5 can be observed in Fig. 6 as a function of the groove profile.

A compression scheme uses several gratings and it would be easier and cheaper to build identical gratings. That requires selection of the profile producing the highest efficiency and the smallest field enhancement. Comparison between Figs. 5(b) and 6(b), however, shows that a groove width $c$ close to $450 \mathrm{~nm}$ produces the smallest enhancement of the electric field inside the solid materials but also, unfortunately, the lowest efficiency so that a choice must be made between efficiency and damage threshold. For a better understanding of this problem, the enhancement of the $|E|^{2}$ is plotted in Fig. 7 as a function of the averaged efficiency. It can be seen that the smallest values of $|E|^{2}$

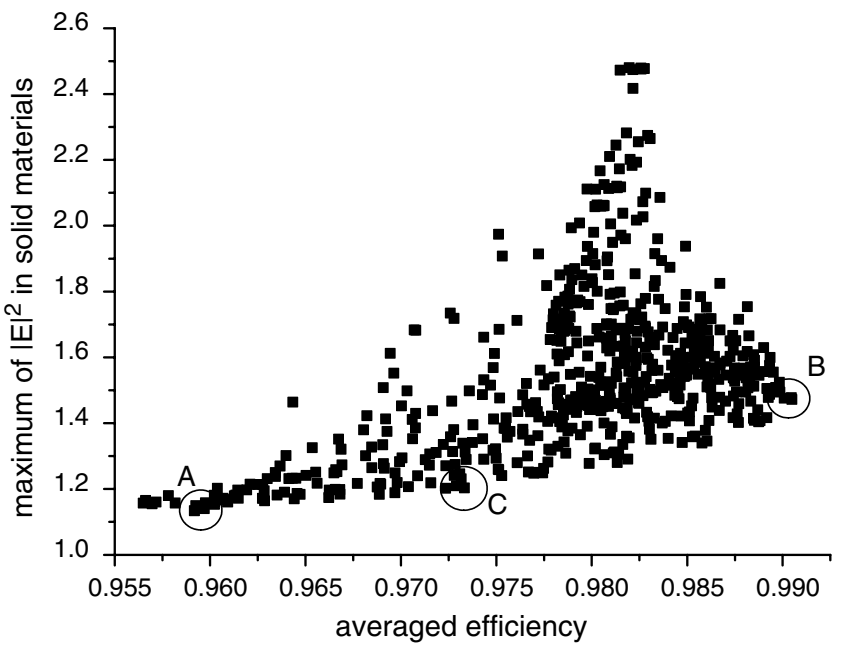

Fig. 7. Maximum of the enhancement of $|E|^{2}$ in the solid materials as a function of the reflected efficiency. An enhancement of about 1.1 can be obtained for efficiencies close to 0.96 (point A) whilst an enhancement 1.5 is observed for the highest efficiencies (point B).

increase with respect to the reflected efficiency. If a maximum of 1.1 of $|E|^{2}$ can be obtained for an efficiency close to 0.96 (point $\mathrm{A}$ ), this maximum value increases up to 1.5 for the highest efficiencies (point B). Thus, it is possible to optimize the first gratings of the compressor system with respect to the efficiency (point A) and the last one with respect to the damage threshold (point B). Another solution may consist of building identical gratings by a tradeoff between the two sets of parameters (point C).

\section{Conclusion}

The aim of this study is to improve the performance levels of gratings used as compressors in high power lasers. In the optimization process, particular attention is paid to the limitations of the fabrication process. The optimization method selects the best profiles in the whole domain of the fabrication fluctuation. Firstly, a numerical optimization of the efficiency in the -1 order in reflection is performed as a function of the thickness of the dielectric layers making up the reflecting stack, and as a function of the grating profile. As far as the mirror is concerned, it has been evidenced that the use of a metal substrate enables a substantial reduction in the number of dielectric bilayers in the stack, and that the mirror stack must be centered at the median angle of the 0 and the -1 orders. As far as the grating profile is concerned, the slope angle must be increased up to $90^{\circ}$ which corresponds to a lamellar profile. Averaged efficiencies over the entire surface of the grating higher than $99 \%$ can be obtained with only 7 bilayers. Secondly, for each selected profile, the maximum of the square of the electric field value is calculated inside the solid material. Plots of these maximums as a function of the grating profile reveal a decrease by a factor close to 2.5 when groove thickness and width values are increased. As a conclusion, among the profiles selected for their good diffrac- 
tion performance levels, those with the highest thickness and width groove values present the smallest enhancement of the electric field norm inside the solid material.

\section{References}

[1] N. Blanchot et al., in: International Conference on Ultrahigh Intensity Lasers, Tahoe City, 2004.

[2] D. Strickland, G. Mourou, Opt. Commun. 56 (1985) 219.

[3] M.D. Perry, R.D. Boyd, J.A. Britten, B.W. Shore, C. Shannon, L. Li, Opt. Lett. 20 (1995) 940.

[4] B.W. Shore, M.D. Perry, J.A. Britten, R.D. Boyd, M.D. Feit, H.T. Nguyen, R. Chow, G.E. Loomis, L. Li, J. Opt. Soc. Am. A 14 (1997) 1224.

[5] H. Wei, L. Li, Appl. Opt. 42 (2003) 6255.

[6] K. Hehl, J. Bischoff, U. Mohaupt, M. Palme, B. Schnabel, L. Wenke, R. Bödefeld, W. Theobald, E. Welsch, R. Sauerbrey, H. Heyer, Appl. Opt. 38 (1999) 6257.
[7] C.P.J. Barty et al., Nucl. Fusion 44 (2004) S266.

[8] B.C. Stuart, M.D. Feit, A.M. Rubenchik, B.W. Shore, M.D. Perry, Phys. Rev. Lett. 74 (1995) 2248.

[9] B. Touzet, J.R. Gilchrist, Photon. Spectra (2003) 67.

[10] H. Leplan, B. Geenen, J.Y. Robic, Y. Pauleau, J. Appl. Phys. 78 (1995) 962.

[11] E.G. Loewen, E. Popov, Diffraction Gratings and Applications, Marcel Dekker, New York, 1997 (Chapter 10).

[12] M. Born, E. Wolf, Principles of Optics, seventh ed., Pergamon, Oxford, UK, 1991

[13] E. Popov, L. Mashev, D. Maystre, Opt. Commun. 65 (1988) 97.

[14] J.R. Marciante, D.H. Raguin, Opt. Lett. 29 (2004) 542.

[15] M. Mero, J. Liu, A. Sabbah, J. Jaspara, K. Starke, D. Ristau, J.K. McIver, W. Rudolph, Laser Induced Damage Opt. Mater. 4932 (2002) 202.

[16] M. Nevière, E. Popov, Light Propagation in Periodic Medias; Differential Theory and Design, Marcel Dekker, New York, Basel, Honk Kong, 2003. 